\title{
MicroRNA 9
}

National Cancer Institute

\section{Source}

National Cancer Institute. MicroRNA 9. NCI Thesaurus. Code C116401.

A 23 ribonucleotide sequence that is a final product of the processing of MIR9-1 premiRNA, MIR9-2 pre-miRNA or MIR9-3 pre-miRNA. This oligonucleotide is involved in gene regulation and may play a role in nerve cell differentiation, insulin secretion and nervous system tumors which display neuronal differentiation. 